International Journal of Pure and Applied Mathematics

Volume 89 No. 1 2013, 55-70

ISSN: 1311-8080 (printed version); ISSN: 1314-3395 (on-line version)

url: http://www.ijpam.eu

doi: http://dx.doi.org/10.12732/ijpam.v89i1.7

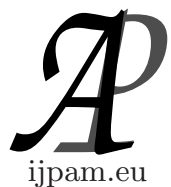

\title{
CONSECUTIVE EVEN NUMBER FINDING GRAPH (CENFG) RELATED TO GOLD BACH CONJECTURE
}

\author{
Rongdeep Pathak ${ }^{1}$, Bichitra Kalita ${ }^{2}$ \\ ${ }^{1,2}$ Department of Computer Application (M.C.A) \\ ${ }^{1,2}$ Assam Engineering College \\ Guwahati, 781013, Assam, INDIA
}

\begin{abstract}
In this paper, the consecutive even number finding graph has been discussed. The solution of Goldbach conjecture has been established from this graph.
\end{abstract}

AMS Subject Classification: 11A41, 11A51, 11A051, 05C30, 05C45 Key Words: the graph CPVEEWGS, CENFG, adjacency matrix

\section{Introduction}

It has been found that Pure Mathematics is mainly associated with abstract concepts. From the eighteenth century onwards, this was a recognized branch of combinatorial activities in various fields such as astronomy, physics, engineering and so on. Finance and cryptography are current example of areas to which pure mathematics is applied in significant ways.

In the branch of Pure Mathematics, the Number theory, which is again playing a very important role in other branches of science and technology occupying a field of conjectural problems. Since the discovery of Number theory it has been occupying a field of so many conjectural problems, which are yet to be proved. There are many questions around Prime numbers, which are remained open, such as Gold Bach's conjecture [1742], which proposes that every even integer greater than 2 can be expressed as sum of two primes, Twin prime

Received: June 18, 2013

(c) 2013 Academic Publications, Ltd.

${ }^{\S}$ Correspondence author url: www.acadpubl.eu 
conjecture which says that there are infinitely many pairs of primes whose difference is 2 and there always a prime between $n^{2}$ and $(n+1)^{2}$ and so on. Many attempts have been made for the proof of these conjectural problems. But, no acceptable solution has been obtained till now or nobody can find a suitable method to carry on this research depending on the available theorems and results.

Comparing the graph theory with number theory, it has been found that the graph theory, which is assumed to be applied, has started before number theory. We know that graph theory started in 1736 from the Euler works on Konigsberg bridge problem. But, interesting relation between graph theory and number theory has not been found except the graphical partition. Hence there is a field of study for existence any important theoretical results between them. Graph theoretical ideas are highly utilized by computer science application such as data mining, database, networking and so on. The concept of graph coloring is utilized in resource allocation and scheduling. Path, walk and circuits in graph theory are used in application of travelling salesman problem, database design concept, networking etc. Here we combine the concept of graph theory and number theory, a branch of pure mathematics for proving the conjectural problem of Gold Bach conjecture. Kaida Shi [13] forwarded a new method to prove Goldbach conjecture and twin prime conjecture. A new direction of proof of Goldbach conjecture has been forwarded by Kalita [4] in 2000. Kalita [5] has also been forwarded the proof of Goldbach conjecture in opposite direction. After the publication of proof of Goldbach conjecture forwarded by Kalita, Alex Vand etal [6] showed that the Goldbach conjecture is true for all the even numbers from 2 to $10^{18}$. Recently Kalita [12] has been forwarded the proof of Goldbach conjecture i.e. every even number $n$ greater than 2 can be expressed as a sum of two primes, with the help of graph theoretic concept. Kalita etal has been discussed some properties of even numbers with various properties of graph and an algorithm has also been forwarded to know the various structure of graphs relating to even numbers $2 \mathrm{n}+4,4 \mathrm{n}+4,(\mathrm{n}+1)(\mathrm{n}+2)$ and $6 \mathrm{n}+$ 2 for $\mathrm{n} \geq 1$.

In this paper, the proof of Goldbach conjecture, which proposes that every even integer greater than 2 can be expressed as sum of two primes, has been forwarded with the help of consecutive even number finding graph (CENFG). Two theorems has been proposed and proved which actually give the new direction of proof of Gold Bach Conjecture. 


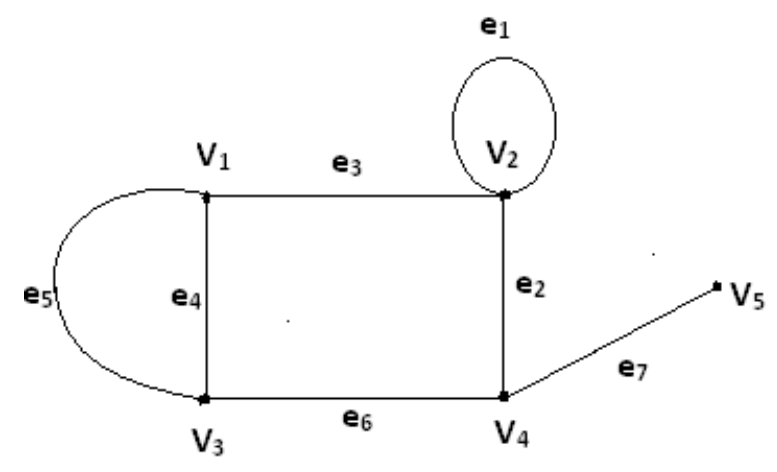

Figure 1

\section{Definitions, Theorems and Algorithm}

Before going to forward the proof of Goldbach conjecture with the help of consecutive even number finding graph, which proposes that every even integer greater than 2 can be expressed as sum of two primes the following definitions and terminologies have been cited to understand the use of graph for Gold Bach conjecture.

Definition 1. A graph $G=(V, E)$ consists of a set of objects $V=\left\{V_{1}, V_{2}\right.$, $\left.\mathrm{V}_{3} \ldots\right\}$ called vertices, and another set $\mathrm{E}=\left\{\mathrm{e}_{1}, \mathrm{e}_{2}, \mathrm{e}_{3}, \ldots\right\}$, whose elements are called edges, such that each edge $e_{k}$ is identified with an unordered pair $\left(v_{i}, v_{j}\right)$ of vertices. The vertices $v_{i}, v_{j}$ associated with edge $e_{k}$ are called end vertices of $e_{\mathrm{k}}$. Graph whose edges or vertices have names are known as labeled. Graph labeling usually refers to the assignment of labels to the edges and vertices of a graph, subject to certain rules depending on the situation.

Definition 2. A graph $\mathrm{G}(\mathrm{V}, \mathrm{E})$, in which there exists an edge between every pair of vertices is called a complete graph.

Definition 3. An edge having the same vertex as both its end vertices is called a self loop.

The graph of five vertices and seven edges with one self loop is shown in Figure 1.

We now remind the following definitions [12] for clarification.

Definition 4. (Prime Vertex and Even Edge Weighted Graph, see [12]) Let $\mathrm{G}(\mathrm{V}, \mathrm{E})$ be a graph having at least one self loop without parallel edges. For the graph $G(V, E)$ we consider the set of vertices $V=\left\{V_{1}, V_{2}, V_{3}, \ldots V_{n}\right\}$ and 


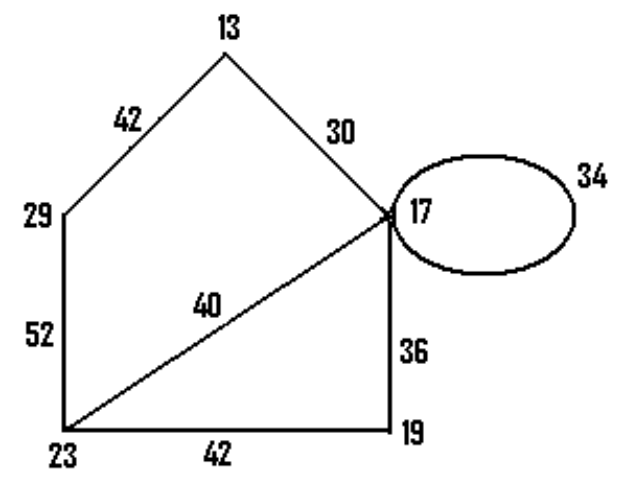

Figure 2

the set of edges $\mathrm{E}=\left\{\mathrm{E}_{1}, \mathrm{E}_{2}, \mathrm{E}_{3}, \ldots . \mathrm{E}_{\mathrm{n}}\right\}$. Let $\mathrm{P}_{1}<\mathrm{P}_{2}<\mathrm{P}_{3}<\ldots<\mathrm{P}_{\mathrm{n}}$ for $\mathrm{n} \geq 5$ be consecutive primes $\geq 13$. Now attaching these primes $\mathrm{P}_{1}<\mathrm{P}_{2}<\mathrm{P}_{3}$ $<\ldots<\mathrm{P}_{\mathrm{n}}$ for $\mathrm{n} \geq 5$ with the vertices $\mathrm{V}_{1}, \mathrm{~V}_{2}, \mathrm{~V}_{3}, \ldots \mathrm{V}_{\mathrm{n}}$ of the graph $\mathrm{G}$ which are now called prime weight $(\mathrm{PW})$ of the vertices and considering weights $\mathrm{W}_{1}$, $\mathrm{W}_{2}, \mathrm{~W}_{3}, \ldots \ldots, \mathrm{W}_{\mathrm{n}}$ for the edges $\mathrm{E}_{1}, \mathrm{E}_{2}, \mathrm{E}_{3}, \ldots . \mathrm{E}_{\mathrm{n}}$, where the weights $\mathrm{W}_{1}$, $\mathrm{W}_{2}, \mathrm{~W}_{3}, \ldots . ., \mathrm{W}_{\mathrm{n}}$ are obtained as a sum of any two prime weights $\mathrm{P}_{\mathrm{i}}+\mathrm{P}_{\mathrm{j}}$ of attached vertices and considering self loop, if exist in the graph as sum of the same prime weight of the attached vertex, we can construct a new graph with prime vertex set $\mathrm{V}_{\mathrm{atP}}$ and the weighted edges set $\mathrm{E}_{\mathrm{soP}}$ and this graph thus obtained is called a prime vertex and even edge weighted graph of the graph $\mathrm{G}(\mathrm{V}, \mathrm{E})$ and it is denoted by the graph PVEEWG $\left(\mathrm{V}_{\mathrm{atP}}, \mathrm{E}_{\mathrm{soP}}\right)$. Figure 2 is a prime vertex and even edge weighted graph $\left(\mathrm{V}_{\mathrm{atP}}, \mathrm{E}_{\mathrm{soP}}\right)$ for the five primes $13<17<19<23<29$. For this graph $\mathrm{V}_{\text {atP }}=\{13,17,19,23,29\}$ and $\mathrm{E}_{\mathrm{soP}}=$ $\left\{\mathrm{W}_{1}=30, \mathrm{~W}_{2}=34, \mathrm{~W}_{3}=36, \mathrm{~W}_{4}=40, \mathrm{~W}_{5}=42, \mathrm{~W}_{6}=42\right.$, $\mathrm{W}_{7}=52$ and the original graph (see Figure 2) has one self loop and this gives an edge of weight $\mathrm{W}_{2}=34$.

Definition 5. (Complete Prime Vertex and Even Edge Weighted Graph, see [12]) A graph PVEEWG ( $\left.\mathrm{V}_{\mathrm{atP}}, \mathrm{E}_{\mathrm{soP}}\right)$ is called a complete prime vertex and even edge weighted graph if it is obtained from a complete graph $\mathrm{G}$ ( V, E ). It is denoted by the graph CPVEEWG $\left(\mathrm{V}_{\mathrm{atP}}, \mathrm{E}_{\mathrm{soP}}\right)$. Figure 3 is a complete prime vertex and even edge weighted graph for the five primes $13<17<19<23$ $<29$.

Definition 6. (Complete Prime Vertex and Even Edge Weighted Graph with Self Loop) Let G (V, E) be a Complete Prime Vertex and Even Edge Weighted graph [12]. We now introduce self loops to all vertices of the graph 


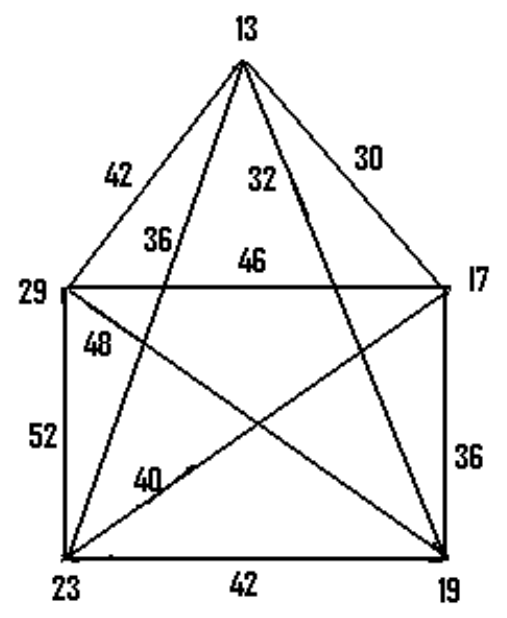

Figure 3

and attached all the consecutive primes to all vertices, where the self loop means addition of same prime for attached weight (Prime) and the edges are represented by the sum of two primes and the graph thus obtained is called Complete Prime Vertex Even Edge Weighted graph with Self loop which is denoted by the graph CPVEEWGS (V, E).

Figure 4 is a complete prime vertex and even edge weighted graph with self loop for the five primes $13<17<19<23<29$.

It is observed that the graph CPVEEWGS (V, E) of Figure 4 always gives even numbers 26, 30, 32, 36, 46, 42, 48, 52, 40, 42, 46, 58, 36, 38, 34 for $\mathrm{n}=5$ consecutive primes, which represents the edges of the CPVEEWGS graph which are actually obtain as a sum of two prime, where $\mathrm{V}$ is the set of vertices 13, 17, 19, 23, 29. But, here, we found that all the even numbers are not consecutive starting from 26 to 58. Interestingly the structure of the graph (CPVEEWGS) is found as a graph CPVEEWGS $\left(\mathrm{X},\left(\mathrm{X}^{2}+\mathrm{X}\right) / 2\right)$, where $\mathrm{X}=5+$ $\mathrm{m}^{*} \mathrm{n}$ for $\mathrm{m}=\mathrm{n}=0,1,2,3$. .

This graph helps us to find out the consecutive even numbers (even weighted edge) up to a certain limit, i.e. the graph of Figure 4 give consecutive even numbers $30,32,34,36,38,40,42$. Here we do not consider the even number 26 for counting the consecutive even numbers as we shall start our investigation from the even number 30 . When we consider the value of $n=6$ consecutive primes, we have the graph CPVEEWGS $\left(\mathrm{X},\left(\mathrm{X}^{2}+\mathrm{X}\right) / 2\right)$ from where the consecutive even numbers (even weighted edges) 30, 32, 34, 36, ..., 62 are to be counted but not starting even number 26. This graph also gives consecutive even numbers 


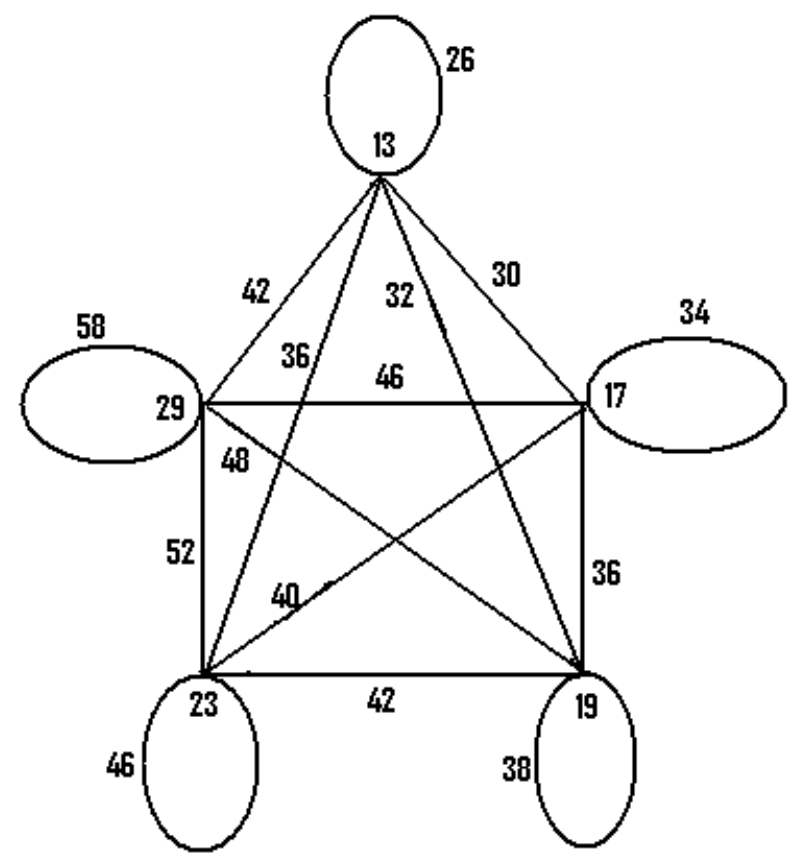

Figure 4

starting from 32 to 54, a total 17 consecutive even numbers.

Observing this formulation of even numbers we have the following important theorem.

Theorem 1. Consecutive even numbers can be calculated out of all even numbers obtained from the graph $G\left(X,\left(X^{2}+X\right) / 2\right)$, where $X=5+m^{*} n$ for $m=n=0,1,2,3$. . up to some certain limit.

Proof. We prove the theorem by induction methods. It is found that the result is true for $\mathrm{X}=5$, that is for $\mathrm{m}=\mathrm{n}=0$. That is when we construct the graph CPVEEWGS (X, $\left.\left(\mathrm{X}^{2}+\mathrm{X}\right) / 2\right)$, for $\mathrm{X}=5$, we have the structure of the graph as shown in Figure 4 from where fifteen even numbers 26, 30, 32, 36, 46, 42, 48, $52,40,42,46,58,36,38,34$ are obtained. Out of these fifteen even numbers we can find seven consecutive even numbers 30, 32, 34, 36, 38, 40, 42 (omitting only 26 as discussed previously). It is observed that for $\mathrm{X}=6$, that is for the values of $\mathrm{m}=\mathrm{n}=1$, we have thirteen consecutive even numbers $30,32,34,36,38$, 40, 42, 44, 46, 48, 50, 52, 54 from the graph CPVEEWGS $(6,21)$. (Note that all the even numbers are nothing but the edges of the graph CPVEEWGS).

Let the theorem be true for $\mathrm{X}=5+\mathrm{k}^{*} \mathrm{k}$ for the values of $\mathrm{m}=\mathrm{n}=\mathrm{k}$. That 


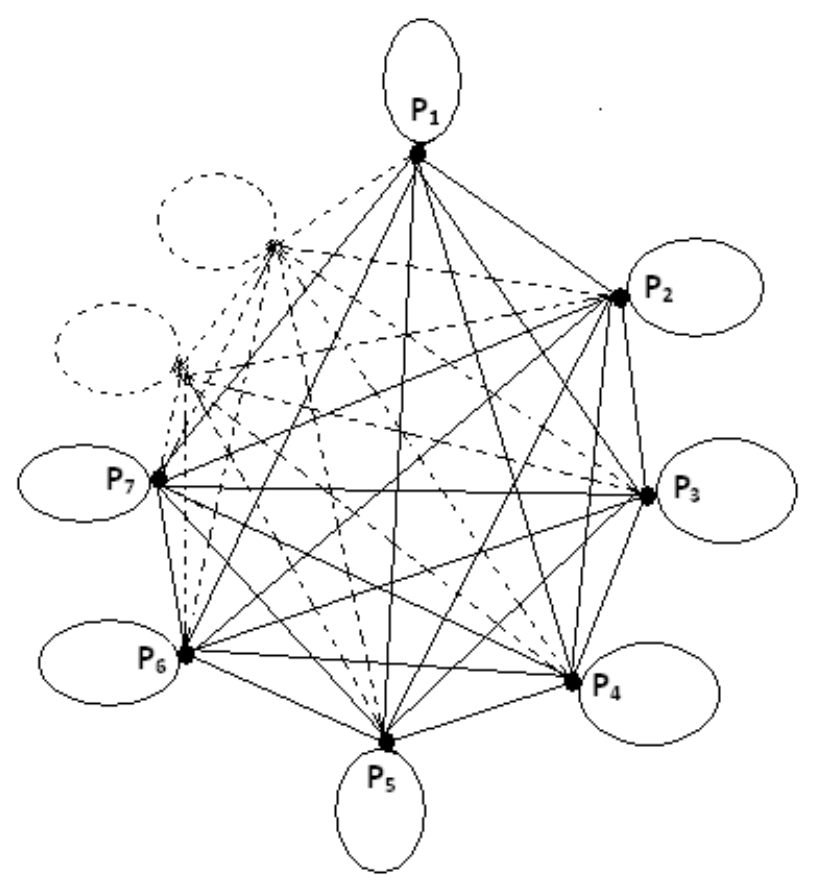

Figure 5

is for the graph CPVEEWGS $\left(5+\mathrm{k}^{*} \mathrm{k},\left(\left(5+\mathrm{k}^{*} \mathrm{k}\right)^{2}+\left(5+\mathrm{k}^{*} \mathrm{k}\right)\right) / 2\right)$, some consecutive even numbers can also be obtained. Now we shall prove it for $\mathrm{X}=$ $5+(\mathrm{k}+1)^{*}(\mathrm{k}+1)$ for the value of $\mathrm{m}=\mathrm{n}=\mathrm{k}+1$. Here the term $\left\{\left(5+(\mathrm{k}+1)^{2}\right.\right.$ \}$\left.^{2}+5+(\mathrm{k}+1)^{2}\right) / 2$ which are the number of even numbers/edges of the graph CPVEEWGS $\left.\left(5+(\mathrm{k}+1)^{2},\left(5+(\mathrm{k}+1)^{2}\right)^{2}+5+(\mathrm{k}+1)^{2}\right) / 2\right)$. It is observed that the result is true for $k=0$, that is the graph CPVEEWGS has six number of vertices when $m=n=1$ and hence our result is true for $m=n=k=1$.According to the definition of the graph CPVEEWGS, the edges represents the even numbers, not all consecutive and from the even numbers and we can calculated some consecutive even numbers. The following graph CPVEEWGS $\left(\mathrm{X},\left(\mathrm{X}^{2}+\mathrm{X}\right) / 2\right)$ for infinite values of $\mathrm{X}$ can be considered for the existence of some consecutive even numbers, where $\mathrm{X}=\left\{\mathrm{P}_{1}, \mathrm{P}_{2}, \mathrm{P}_{3}, \ldots \ldots \ldots\right\}$ and $\left(\mathrm{X}^{2}+\mathrm{X}\right) / 2=\left\{\mathrm{P}_{1}+\mathrm{P}_{2}\right.$, $\mathrm{P}_{1}+\mathrm{P}_{3}, \ldots \ldots$ with $\left.\mathrm{P}_{1}+\mathrm{P}_{1}, \mathrm{P}_{2}+\mathrm{P}_{2}, \ldots, \mathrm{P}_{\mathrm{n}}+\mathrm{P}_{\mathrm{n}}, \ldots \ldots\right\}$ are the edges of the complete graph with self loop.

The graph CPVEEWGS $\left(\mathrm{X},\left(\mathrm{X}^{2}+\mathrm{X}\right) / 2\right)$, gives some even numbers and out of these some even number, we can calculate the consecutive even numbers up to a certain limit. 
Hence the theorem i sproved.

The above theorem gives the information of even numbers/edges from where certain consecutive even numbers can be calculated which are nothing but sum of two primes and hence we require some other method from where one can find out the all consecutive even numbers/edges. Here, we use one important algorithm to get one adjacent matrix from where another structure of graph called consecutive even number finding graph (CENFG), can be constructed which will be discussed later.

Let us first develop the following algorithm.

Algorithm 1. Construction of adjacent matrix for even number Finding graph (CENFG)

INPUT: Number of prime, N

OUTPUT: Find the adjacency matrix with self loop for CENFG

Step 1. Store $\mathrm{N}$ consecutive prime numbers $\left(\mathrm{P}_{1}<\mathrm{P}_{2}<\mathrm{P}_{3}<\ldots<\mathrm{P}_{\mathrm{N}}\right)$ in an array $\mathrm{A}[]$ of size $\mathrm{N}$, where $\mathrm{P}_{1}=13$ and $\mathrm{N} \geq 5$.

Step 2. Calculate the sum of two prime numbers and result store in a $2 \mathrm{D}$ array $\mathrm{B}[][]$ of size $\mathrm{N}^{*} \mathrm{~N}$.

1. Repeat for $\mathrm{I}=1$ to $\mathrm{N}$

2. Begin

3. Repeat for $\mathrm{J}=1$ to $\mathrm{N}$

4. Begin

5. $\mathrm{B}[\mathrm{I}][\mathrm{J}]=\mathrm{A}[\mathrm{I}]+\mathrm{A}[\mathrm{J}]$

6. End

7. End

Step 3. Store the values of the $2 \mathrm{D}$ array $\mathrm{B}[\mathrm{]}[\mathrm{in}$ to an another array $\mathrm{C}[]$ of size $\mathrm{N}^{*} \mathrm{~N}$

1. Repeat for $\mathrm{I}=1$ to $\mathrm{N}$

2. Begin

3. Repeat for $\mathrm{J}=1$ to $\mathrm{N}$

4. Begin 
5. $\mathrm{C}[\mathrm{K}]=\mathrm{B}[\mathrm{I}][\mathrm{J}]$

6. $\mathrm{K}=\mathrm{K}+1$

(Initial value of $\mathrm{K}$ is 0 )

g. End

h. End

Step 4. Sorting the value of $\mathrm{C}\left[\mathrm{N}^{*} \mathrm{~N}\right]$ in an ascending order.

1. Repeat for $\mathrm{I}=1$ to $\left(\mathrm{N}^{*} \mathrm{~N}-1\right)$

2. Begin

3. Repeat for $\mathrm{J}=(\mathrm{I}+1)$ to $\mathrm{N}^{*} \mathrm{~N}$

4. Begin

5. If $(\mathrm{C}[\mathrm{I}]>\mathrm{C}[\mathrm{J}])$

6. Temp1 $=\mathrm{C}[\mathrm{I}]$

7. $\mathrm{C}[\mathrm{I}]=\mathrm{C}[\mathrm{J}]$

8. $\mathrm{C}[\mathrm{J}]=$ Temp1

9. End

10. End

Step 5. Remove duplicate values from $\mathrm{C}\left[\mathrm{N}^{*} \mathrm{~N}\right]$.

1. $\mathrm{M}=\mathrm{N}^{*} \mathrm{~N}$

2. Repeat for $I=1$ to $M-1$

3. Begin

4. Repeat for $\mathrm{J}=\mathrm{I}+1$ to $\mathrm{M}$

5. Begin

6. If ( $\mathrm{C}[\mathrm{I}]=\mathrm{C}[\mathrm{J}])$

7. $\mathrm{M}=\mathrm{M}-1$

8. Repeat for $\mathrm{K}=\mathrm{J}$ to $\mathrm{M}$ 
9. Begin

10. $\mathrm{C}[\mathrm{K}]=\mathrm{C}[\mathrm{K}+1]$

11. End

12. $\mathrm{J}=\mathrm{J}-1$

13. End

14. End

Step 6. Except the first set of consecutive even numbers of the array $\mathrm{C}\left[\mathrm{N}^{*} \mathrm{~N}\right]$ (not considering the first value i.e. 26) change the values of the remaining even numbers to zero.

Step 7. Largest value of $\mathrm{C}\left[\mathrm{N}^{*} \mathrm{~N}\right]$ is stored in TEMP value.

Step 8. Change the values of $\mathrm{B}[][]$ to zero which are larger than TEMP

1. Repeat for $\mathrm{I}=1$ to $\mathrm{N}$

2. Begin

3. Repeat for $\mathrm{J}=1$ to $\mathrm{N}$

4. Begin

5. If ( $\mathrm{B}[\mathrm{I}][\mathrm{J}]>\mathrm{TEMP})$

6. $\mathrm{B}[\mathrm{I}][\mathrm{J}]=0$

7. End

8. End

Step 9. Construct an intermediate matrix from which one can finally develop an adjacency matrix.

1. Initialize $\mathrm{B}[1][1]=0$

2. Repeat for $I=1$ to $\mathrm{N}$

3. Begin

4. Repeat for $\mathrm{J}=1$ to $\mathrm{N}$ 
5. Begin

6. If $\mathrm{B}[\mathrm{I}][\mathrm{J}]==0$ go to step 9.d

7. Repeat for $\mathrm{X}=0$ to $\mathrm{N}$

8. Begin

9. Repeat for $\mathrm{Y}=0$ to $\mathrm{N}$

10. Begin

11. If $(\mathrm{I}==\mathrm{X} \& \& \mathrm{~J}==\mathrm{Y})$

12. Go to step $9 . \mathrm{i}$

13. Else

14. If $(\mathrm{B}[\mathrm{I}][\mathrm{J}]==\mathrm{B}[\mathrm{X}][\mathrm{Y}] \& \&(\mathrm{I}+\mathrm{X}) \neq(\mathrm{J}+\mathrm{Y}))$

15. $\mathrm{B}[\mathrm{X}][\mathrm{Y}]=0$

16. End

17. End

18. End

19. End

Step10. Construct the final Matrix from which we can draw Consecutive Even Number Finding (CENFG) graph.

1. Repeat for $\mathrm{I}=1$ to $\mathrm{N}$

(a) Begin

i. Repeat for $\mathrm{J}=0$ to $\mathrm{N}$

ii. Begin

iii. If $(\mathrm{B}[\mathrm{I}][\mathrm{J}] \geq 1)$

iv. $\mathrm{B}[\mathrm{I}][\mathrm{J}]=1$

v. End

(b) End 


\begin{tabular}{|l|l|l|l|l|l|l|}
\hline & $\mathbf{P}_{\mathbf{1}}=13$ & $\mathbf{P}_{\mathbf{2}}=17$ & $\mathbf{P}_{\mathbf{3}}=19$ & $\mathbf{P}_{\mathbf{4}}=23$ & $\mathbf{P}_{\mathbf{5}}=29$ & $\mathbf{P}_{\mathbf{6}}=31$ \\
\hline $\mathbf{P}_{\mathbf{1}}=13$ & 0 & 1 & 1 & 1 & 1 & 1 \\
\hline $\mathbf{P}_{\mathbf{2}}=17$ & 1 & 1 & 0 & 1 & 1 & 1 \\
\hline $\mathbf{P}_{\mathbf{3}}=19$ & 1 & 0 & 1 & 0 & 0 & 1 \\
\hline $\mathbf{P}_{\mathbf{4}}=23$ & 1 & 1 & 0 & 0 & 1 & 1 \\
\hline $\mathbf{P}_{\mathbf{5}}=29$ & 1 & 1 & 0 & 1 & 0 & 0 \\
\hline $\mathbf{P}_{\mathbf{6}}=31$ & 1 & 1 & 1 & 1 & 0 & 0 \\
\hline
\end{tabular}

Table 1

\section{Step 11. STOP}

From the algorithm, we can find an adjacency matrix which is nothing but a representation of vertices/primes and the interconnection between vertices/primes. This adjacency matrix gives the total number of edges of the graph CENFG (V, E), which are consecutive even numbers which are actually found as a sum of two primes. The number of edges $(\mathrm{P})$, which is found from the adjacency matrix always greater than the value of $\mathrm{N}$, which is the input to the algorithm. Here, $\mathrm{N}$ is the number of consecutive primes/vertices of the graph CENFG (X, P), which can be drawn from the adjacency matrix.

Here let us take one example for $\mathrm{N}=6$, say for six consecutive primes starting from 13 .

After applying the algorithm, we have the following table (1) which gives the adjacency matrix with self loop obtained from the algorithm from which we can draw the consecutive even number finding graph CENFG (V, E) as shown in Figure 6.

The following graph of Figure 6 is a consecutive even number finding graph (CENFG) which is drawn with the help of table 1 and from this graph one can find out the consecutive even numbers which are obtained as a sum of two primes/vertices.

The above graph CENFG $(6,13)$ gives thirteen consecutive even numbers /edges which are obtained as a sum of two primes/vertices and this gives the proof of Goldbach conjecture for thirteen consecutive even numbers which can be expressed as a sum of two primes. Hence the following theorem can be considered for counting all consecutive even numbers/edges.

We can now define the consecutive even number finding graph as follows:

Definition 7. (Consecutive Even Number Finding Graph) A prime vertex and even edge weighted graph $[12] \mathrm{G}(\mathrm{V}, \mathrm{E})$ is called a consecutive even number 


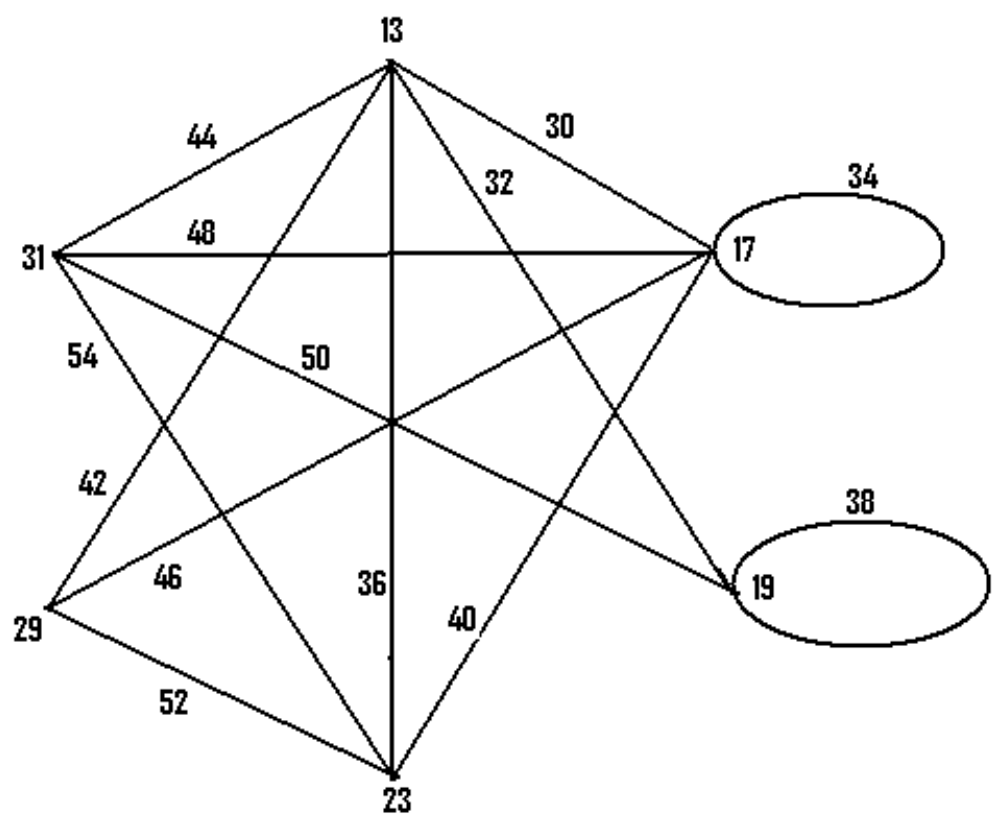

Figure 6

finding graph if all the edges of the graph gives consecutive even numbers and this graph is denoted as a graph CENFG (V, E) where $\mathrm{V}$ is the number of attached vertices and $\mathrm{E}$ is the number of edges/even number.

Figure 7 is a graph CENFG $(21,76)$. This graph gives seventy six consecutive even numbers/edges, starting from thirty to one hundred eighty, which are obtained from the algorithm.

Theorem 2. The graph CENFG $(X, P)$, where $X=5+m^{*} n, m=n=0,1,2$, 3 ... and $P$ is the value that can be calculated from the matrix obtained from the algorithm, always gives consecutive even numbers/edges which are the sum of two primes.

Proof. It is found from the Algorithm 1 that $\mathrm{P}$ is the number of edges which can be calculated from the adjacency matrix and $\mathrm{P}$ always represents consecutive even numbers starting from thirty.

Now, if we put the value of $\mathrm{X}=6$, that is $\mathrm{m}=\mathrm{n}=1$, we can find an adjacency matrix as shown in table 1 and from this table, we can draw a graph CENFG $(6,13)$ as shown in Figure 6 . This graph gives thirteen even weighted edges/ numbers, which are 30, 32, 34, 36, 38, 40, 42, 44, 46, 48, 50, 52 and 54 .

Continuing the process of construction of the graph CENFG for $\mathrm{X} \geq 6$ 


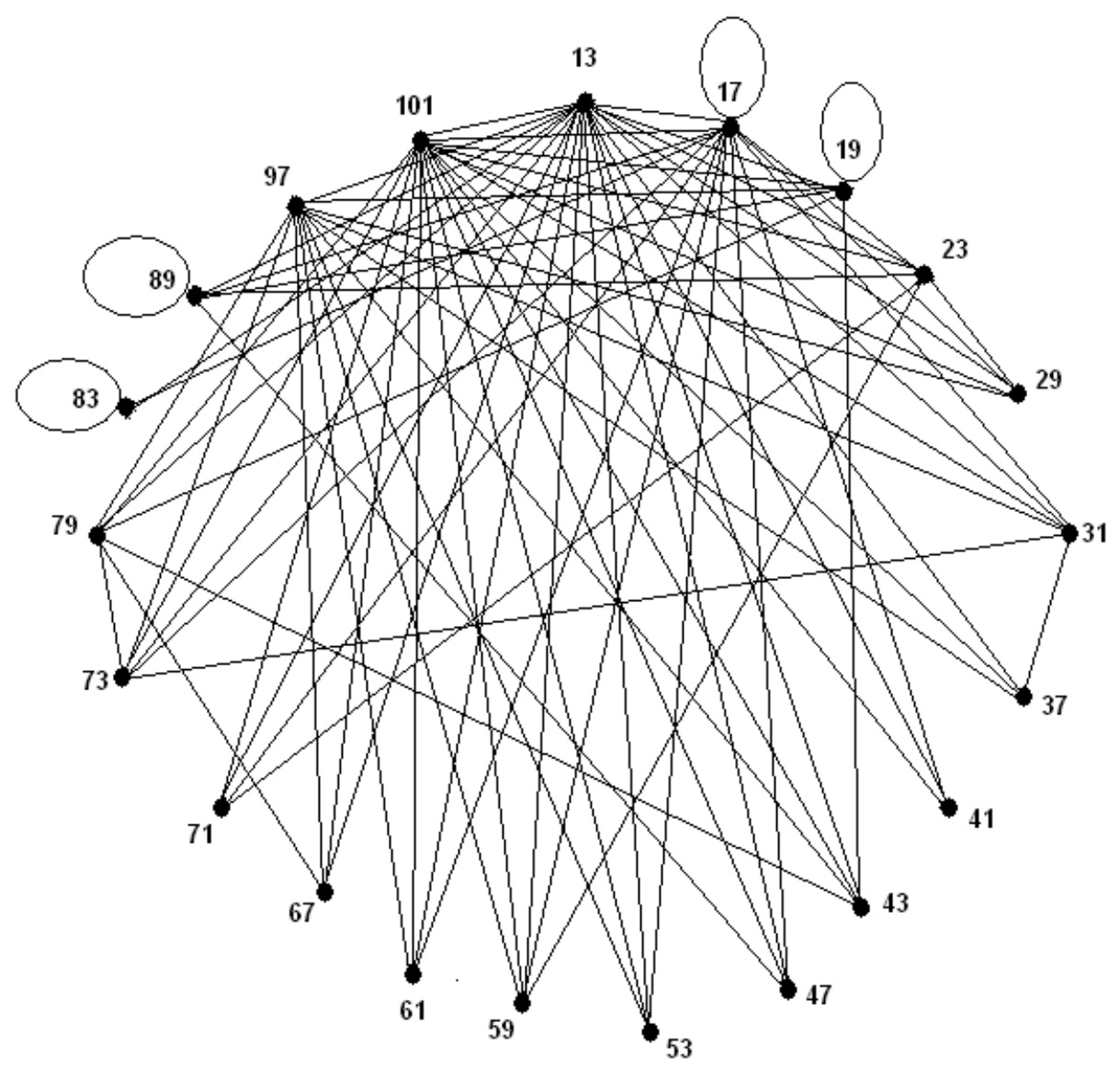

Figure 7

and $\mathrm{m}=\mathrm{n} \geq 2$, we can have the consecutive even numbers /edges to any limit. For example, if we put the value of $\mathrm{X}=21$, that is $\mathrm{m}=\mathrm{n}=4$, we can find another graph CENFG $(21,76)$ as shown in Figure 7. This graph CENFG $(21,76)$ gives seventy six even weighted edges/ numbers starting from thirty to one hundred eighty.

We see that, the number of even weighted edges/numbers gradually increasing when the value of $\mathrm{X}$ increases. Again it is found from the algorithm that the value of $\mathrm{P}$, which is the number of even weighted edges/numbers always greater than the value of $\mathrm{N}$, which is the number of vertices/primes.

Hence, the graph CENFG (X, P), always gives consecutive even numbers/edges, which are the sum of two primes/vertices. 
Gold Bach Conjecture. Every even number greater than 2 can be expressed as a sum of two primes.

Proof. From the theorem 2, we can find that the graph CENFG (X, P), gives infinite consecutive even numbers/edges that is values of $\mathrm{P}$ for infinite value of $X$. This immediately satisfies that every even number greater than 30 can be expressed as sum of two primes, which completes the proof of Gold Bach Conjecture.

Note. Here we should not consider the smaller values of even number from 2 to 28 as they are easy to express as a sum of two primes.

\section{Conclusion}

In this paper, the proof of Gold bach conjecture, which proposes that every even integer greater than 2 can be expressed as sum of two primes, has been forwarded with the help of consecutive even number finding graph (CENFG). Here, the solution of Gold bach conjecture is established with the help of graph theory.

\section{References}

[1] Richard Courant, H. Robbins, What is Mathematics, Oxford University Press (1996).

[2] G.P. Loweke, The Lour of Prime Numbers, Vontage Press, N.Y. (1982).

[3] F. Harary, Graph Theory, Narosa Publishing House.

[4] B. Kalita, Some conjectural problems of primes and partial solution of it, In: International Conferences on Recent Advances in Mathematical Sciences, I.I.T Kharagpur (2000).

[5] B. Kalita, Proof of Goldbach conjecture and related conjecture of primes, Bull. Pure and Applied Sciences, 25E, No. 2 (2006), 451-459.

[6] V. Alex, et al, Goldbach Suspects a Step Further, Sources Kennislink, Language, Dutch, On-line article (2007).

[7] B. Kalita, Certain forms of even numbers and their properties, Global Journal of Pure and Applied Mathematics, 5, No. 1 (2009), 53-58. 
[8] B. Kalita, et al, Some properties of graphical partitions, Int. Journal of Appl. Engineering Research, 4, No. 10 (2009), 1851-1856.

[9] C. Pomerance, The Search for Prime Numbers, Scientific American (1982).

[10] B. Kalita, A new set of non planar graphs, Bull. Pure and Applied Sci., 24E, No. 1 (2005), 29-38.

[11] B. Kalita, Some Investigations on Graph Theory, Ph.D. Thesis, Finance India, XIX, No. 4 (2005), 1430-1438.

[12] B. Kalita, Graph and Goldbach conjecture, International Journal of Pure and Applied Mathematics, 82, No. 4 (2013), 531-546.

[13] Kaida Shi, A new method to proof Goldbach conjecture, Twin prime conjecture and other two propositions, Cornell University Library, ArXiv: math/0309103v1 (2003). 